\title{
A survey on acute kidney injury in severely and critically ill COVID-19 patients without chronic kidney disease
}

\author{
Yue Yu\#, Huipeng Ge\#, Xiufen Wang", Zhonghua Huang, Lei Chen, Yun Han, Dayang Jiang, Lijian Tao, \\ Qiaoling Zhou, Zhangzhe Peng, Xiangcheng Xiao \\ Department of Nephrology, Xiangya Hospital, Central South University, Changsha, China \\ Contributions: (I) Conception and design: Y Yu, H Ge, Z Peng; (II) Administrative support: Y Yu, H Ge, X Wang, Z Peng, X Xiao; (III) Provision of \\ study materials or patients: Y Yu, H Ge; (IV) Collection and assembly of data: Y Yu, H Ge, X Wang; (V) Data analysis and interpretation: H Ge; (VI) \\ Manuscript writing: All authors; (VII) Final approval of manuscript: All authors. \\ "These authors contributed equally to this work. \\ Correspondence to: Zhangzhe Peng; Xiangcheng Xiao. Department of Nephrology, Xiangya Hospital, Central South University, 87 Xiangya Road, \\ Changsha 410008, China. Email: pengzhangzhe@csu.edu.cn; xiaoxc@csu.edu.cn.
}

\begin{abstract}
Background: Research has shown that acute kidney injury (AKI) has a noticeable incidence in critically ill patients with coronavirus disease 2019 (COVID-19). Patients with prior renal insufficiency are particularly susceptible to severe acute respiratory syndrome coronavirus-2 (SARS-CoV-2), due to their immune dysfunction. However, most patients with COVID-19 do not have a history of kidney dysfunction, and few studies have focused on the incidence of AKI among COVID-19 patients without chronic kidney disease (CKD). In this study, we aimed to investigate the occurrence of AKI in severely and critically ill COVID-19 patients, with a particular focus on those without a CKD history.
\end{abstract}

Methods: A single-center retrospective study of 96 patients with COVID-19 in China between February 7 and March 3, 2020 was conducted. All patients were diagnosed by nucleic acid test (NAT) for SARS-CoV-2. Enrolled patients were divided into the severely or critically ill group according to the defined criteria. Patients' epidemiological, clinical, and laboratory characteristics, along with their treatment information, were collected from the medical history system. The occurrence of AKI was compared between the severe and critical patients, and between patients with or without a history of CKD. The diagnostic criteria for AKI included an increase in the serum creatinine level to $\geq 1.5$-fold the level at baseline within 7 days according to the Kidney Disease: Improving Global Outcomes (KDIGO) guidelines. Renal outcomes were defined as AKI or non-AKI.

Results: Four patients (4.2\%) developed AKI, all of whom were in the critically ill group, and 3 (75\%) of whom died. Out of the 90 severely and critically ill COVID-19 patients without CKD, 3 (3.3\%) patients developed AKI; out of the 6 patients with CKD, 1 (16.7\%) patient developed AKI. Age, disease severity, procalcitonin, C-reactive protein, and interleukin-6 were correlated with AKI onset in severely and critically ill COVID-19 patients, while lymphocyte count and estimated glomerular filtration rate at admission were inversely related to the development of AKI.

Conclusions: Only 3.3\% of severely and critically ill COVID-19 patients without CKD in our research cohort developed AKI. Critically ill patients may be more susceptible to AKI than severely ill patients.

Keywords: Acute kidney injury (AKI); coronavirus disease 2019 (COVID-19); renal prognosis; severe acute respiratory syndrome coronavirus-2 (SARS-CoV-2); treatment

Submitted Nov 02, 2020. Accepted for publication Apr 20, 2021.

doi: 10.21037/apm-20-2170

View this article at: http://dx.doi.org/10.21037/apm-20-2170 


\section{Introduction}

With the battle against the life-threatening coronavirus disease 2019 (COVID-19) continuing worldwide, humankind is facing an immense challenge. The virus responsible for COVID-19, severe acute respiratory syndrome coronavirus-2 (SARS-CoV-2), is mostly spread through person-to-person transmission, although its source remains unknown (1). Clinical manifestations of COVID-19 include fever, dry cough, fatigue, diarrhea, expectoration, polypnea, dyspnea, headache, pharyngalgia, and sore muscles $(2,3)$. Given that the kidneys serve as important metabolic organs and are target organs for SARS-CoV-2 to attack (4), the relationship between COVID-19 and acute kidney injury (AKI) is receiving increasing attention $(4,5)$. A retrospective study reported that renal impairment is common in patients with COVID-19 and may be among the major causes of multiorgan failure and death (4), furthermore, AKI has been reported to occur in $5.1 \%$ of patients, with kidney disease being associated with inhospital death. However, most patients in the study had intrinsic kidney damage (with $43.9 \%$ of patients having proteinuria and $26.7 \%$ having hematuria before admission). Consistent with this, a recent article showed that AKI is common in critically ill patients with COVID-19 and prior renal insufficiency (6). However, it should be noted that individuals without kidney disease comprise the majority of COVID-19 patients, although few studies have focused on the incidence of AKI in these patients. How COVID-19 influences the renal function of severely and critically ill patients without a history of chronic kidney disease (CKD) remains unclear.

In this survey, we investigated the incidence of AKI in patients with COVID-19 to determine whether patients without a history of CKD are less susceptible to developing AKI than those with prior CKD. Further, as the identification of effective treatments for COVID-19 is of critical importance at present (2,3), we also summarized the treatments of COVID-19 patients, with a particular focus on those with AKI onset.

We present the study in accordance with the STROBE reporting checklist (available at http://dx.doi.org/10.21037/ apm-20-2170).

\section{Methods}

This study was approved by the ethics committee of Xiangya Hospital, China (No. 202003049), and was in adherence with the Declaration of Helsinki (as was revised in 2013). Informed consent was obtained from the patients before data collection.

\section{Patients}

In this study, 96 adults who were diagnosed with COVID-19 and admitted to the Union Hospital (Wuhan, China) between February 7 and March 3, 2020 were enrolled. The inclusion criteria were severely ill or critically ill patients with a positive nucleic acid test (NAT) for SARSCoV-2. Samples were obtained at admission and every other day after clinical admission for re-examination. All patients had a definite outcome (discharge or death).

\section{Study design and settings}

The demographic data of the 96 patients were collected from the medical history system. These data included basic information (sex, age, days from symptoms to admission, hospital stay, and comorbidities), signs and symptoms (e.g., fever, dyspnea, dry cough, fatigue, diarrhea, expectoration, myalgia, chest distress, headache, nausea, and pharyngalgia), and laboratory findings (blood routine, urine analysis, blood biochemistry, coagulation function, cytokines, and lymphocyte subsets). The estimated glomerular filtration rate (eGFR) was calculated by applying the Chronic Kidney Disease Epidemiology Collaboration (CKD-EPI) equation (7). According to the Kidney Disease: Improving Global Outcomes (KDIGO) guidelines (8), the diagnostic criteria for AKI included an increase in the serum creatinine level to $\geq 1.5$-fold the level at baseline within 7 days (baseline creatinine was defined as the lowest level of creatinine detected during hospitalization).

The characteristics of severe cases and critical cases were compared. Severely ill patients were defined as those meeting any of the following conditions: polypnea with a respiratory rate of over 30 times per minute; oxyhemoglobin saturation $\leq 93 \% ; \mathrm{PaO}_{2} / \mathrm{FiO}_{2} \leq 300 \mathrm{mmHg}(1 \mathrm{mmHg}=0.133 \mathrm{kPa})$; and pulmonary imaging showing obvious progression of lesions $>50 \%$ within $24-48$ hours. Critically ill patients were defined as those meeting any of the following conditions: respiratory failure with mechanical ventilation; shock; other organ failure; and admission to the intensive care unit (ICU). The criteria for discharge were: the absence of fever for at least 3 days; a substantial improvement in both lungs on chest computed tomography (CT); clinical remission of 
Table 1 Treatment regimen

\begin{tabular}{|c|c|c|c|c|}
\hline Variable & Total $(n=96), n(\%)$ & Severely ill ( $n=61), n(\%)$ & Critically ill ( $n=35), n(\%)$ & $P$ value \\
\hline Anticoagulation therapy & $18(18.8)$ & $9(14.8)$ & $9(25.7)$ & 0.185 \\
\hline Antibiotic treatment & $49(51.0)$ & $25(41.0)$ & $24(58.6)$ & $0.009^{* *}$ \\
\hline Antifungal treatment ${ }^{a}$ & $5(5.2)$ & $0(0)$ & $5(14.3)$ & $0.005^{\star *}$ \\
\hline Intravenous immunoglobulin therapy & $3(3.1)$ & $1(1.6)$ & $2(5.7)$ & 0.552 \\
\hline $\mathrm{CRRT}^{\mathrm{a}}$ & $4(4.2)$ & $0(0.0)$ & $4(11.4)$ & $0.016^{\star}$ \\
\hline Traditional Chinese medicine ${ }^{a}$ & 87 (90.6) & $53(86.9)$ & $34(97.1)$ & 0.149 \\
\hline Antiviral therapy & 90 & & & \\
\hline Oseltamivir $^{\mathrm{a}}(75 \mathrm{mg} / \mathrm{d})$ & $4(4.2)$ & $1(1.6)$ & $3(8.6)$ & 0.136 \\
\hline
\end{tabular}

${ }^{a}$ Using the fisher exact test; ${ }^{*} \mathrm{P}<0.05$; ${ }^{*} \mathrm{P} \leq 0.01$. CRRT, continuous renal replacement therapy.

respiratory symptoms; and negative throat swab samples for SARS-CoV-2 twice (for both RNA and antibodies) obtained at least 24 hours apart. A false negative outcome was defined as a negative result followed by a positive result for the same patient during hospitalization.

\section{Treatment}

All patients were treated from the day of admission. Depending on the patient's medical condition, oxygen therapy, traditional Chinese medicine (including Lianhuaqingwen capsules and other Chinese herbs), antiviral therapy (arbidol, ribavirin, and oseltamivir), anticoagulation therapy (heparin sodium), antibiotics (moxifloxacin, meropenem, piperacillin, and linezolid), anti-fungal treatment (caspofungin), glucocorticoids (methylprednisolone), or intravenous immunoglobulin therapy were given (Table 1).

\section{Statistical analysis}

The Kolmogorov-Smirnov test was used under the assumption of normality. Results for categorical variables were expressed as frequencies and percentages. Normally distributed continuous variables were expressed as the mean \pm standard deviation (SD), while the median and interquartile range (IQR) were used for non-normally distributed variables. Categorical variables were compared using the chi-square or Fisher's exact test. Continuous variables were compared using the Student's $t$-test or MannWhitney U test. Spearman's correlation analysis was used to analyze AKI-related variables. $\mathrm{P}<0.05$ was considered to be statistically significant. Statistical analyses were performed using SPSS version 22.0 software (IBM).

\section{Results}

\section{Baseline characteristics}

In this study, 96 patients ( 35 critically ill patients and 61 severely ill patients) were enrolled. The average age of the study population was 63 years, and males comprised $58.3 \%$ of the study population. The median time from disease onset to admission was 13 days (IQR, 10-16 days). The average length of hospital stay was 35 [22-43] days. Among the study population, $58.3 \%(56 / 96)$ of patients had at least 1 comorbidity, among which hypertension (34/96) was the most common, closely followed by diabetes (20/96). Respiratory disease, digestive system disease, and CKD were less common in the patient population.

Regarding the vital signs of patients at admission, the body temperature was $36.7^{\circ} \mathrm{C}\left(\mathrm{IQR}, 36.3-37.1{ }^{\circ} \mathrm{C}\right)$, which might be associated with pre-hospital anti-febrile treatment. The median heart rate and respiratory rate were $88 / \mathrm{min}$ (IQR, 79-100) and 20/min (IQR, 20-24), respectively. 
Table 2 Baseline characteristics

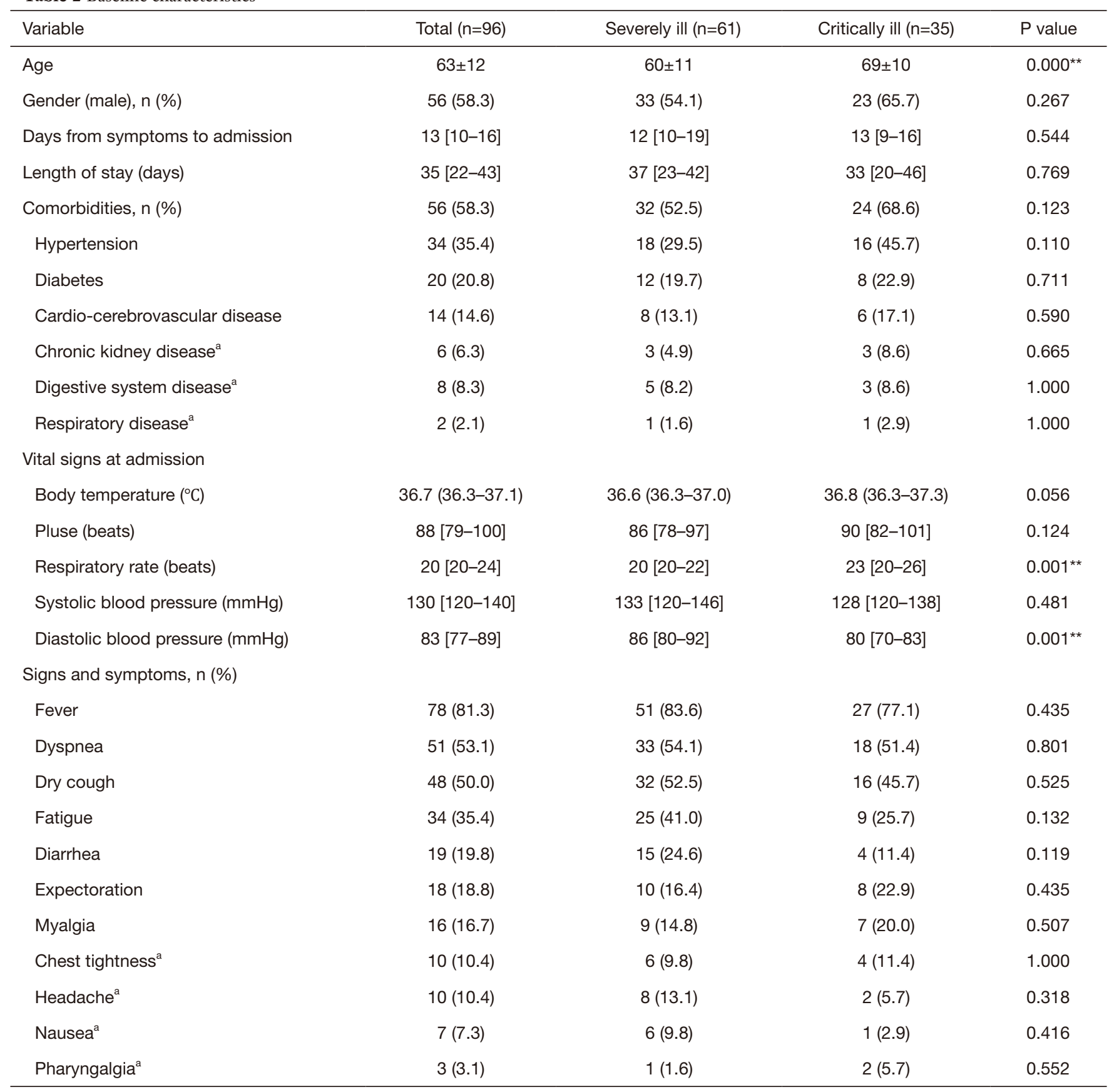

${ }^{a}$ Using fisher exact test; ${ }^{\star \star} \mathrm{P} \leq 0.01$. The data are shown as frequencies and percentages, means \pm standard deviation, or medians with interquartile ranges.

Further, the median systolic and diastolic blood pressure were $130 \mathrm{mmHg}$ (IQR, $120-140 \mathrm{mmHg}$ ) and $83 \mathrm{mmHg}$ (IQR, 77-89 $\mathrm{mmHg}$ ), respectively. Symptoms at disease onset included fever (78/96), dyspnea (51/96), dry cough (48/96), fatigue (34/96), diarrhea (19/96), and expectoration
(18/96); less common symptoms included myalgia, chest tightness, headache, nausea, and pharyngalgia (Table 2).

Patients in the critically ill group were older $(69 \pm 10$ vs. $60 \pm 11$ ), and had a higher respiratory rate and lower diastolic blood pressure at admission than the severely ill 
patients. However, no significant differences were observed in comorbidities or symptoms between the two groups.

\section{Laboratory results}

Compared with severely ill patients, critically ill patients were more likely to have a lower lymphocyte count, lower albumin level, and lower levels of high density lipoprotein (HDL) and hyponatremia. Moreover, critically ill patients had significantly higher levels of D-dimers, a longer prothrombin time, a longer activated partial thromboplastin time, and higher levels of fibrinogen, aspartate aminotransferase, lactate dehydrogenase, and a-hydroxybutyrate dehydrogenase than did severely ill patients. Moreover, critically ill patients showed decreased lymphocytes, increased AST levels, and increased LDH levels compared to severely ill patients. In terms of cytokine levels, in 42 critically ill patients the level of interleukin (IL)-6 was significantly higher than that of 26 severely ill patients. However, no differences were observed in the levels of IL-2, IL-4, interferon (IFN)- $\alpha$, or IFN- $\gamma$ between the two groups. Notably, 67 of 68 patients had an elevated IL-6 level and approximately one-third of the 68 patients had increased IL-2, IL-4, and IL-10 levels. Except for the percentage of B lymphocytes, there were no differences in lymphocyte subsets between the two groups. Indicators that reflect inflammation, such as procalcitonin (PCT) and C-reactive protein (CRP), were also significantly increased in critically ill patients compared to severely ill patients (Table 3).

In this study, we also focused on the detection methods of COVID-19. All patients underwent laboratory tests combined with NAT by throat swab and ORF1ab serum antibody detection. Except for 1 patient, who did not obtain a negative test result, 95 patients were SARS-CoV-2 negative before death or discharge. The false-negative rate of the combined testing method was almost one third. The average duration from the first positive SARS-CoV-2 result to a negative result was 17 days (IQR, 13-22 days). Also, the duration from disease onset to viral shedding was 27 days (IQR, 23-34 days). No statistical differences were observed between the critically ill patients and severely ill patients in terms of the negative conversion rate, false-negative rate, or viral shedding duration (Table 4).

\section{The prevalence of AKI and outcomes in the study population}

In our study, 4 patients (4.2\%) were diagnosed with AKI, all of whom were in the critically ill group. One (16.7\%) patient developed AKI out of the 6 patients with a CKD history, while 3 (3.3\%) patients developed AKI out of the 90 patients without a CKD history. Of the 3 patients, 1 patient had proteinuria, 1 had hematuria, and 1 had both proteinuria and hematuria. Our data showed that 6 patients (6.3\%) died during hospitalization, with 90 patients (93.7\%) being discharged. Of the 6 patients who died, 3 patients had AKI. Because of the small sample size, we could not explore the relationship between AKI and mortality. Furthermore, at admission, the eGFR did not differ significantly between the two groups. Spearman's correlation analysis showed that age, the patient's condition, PCT, CRP, and IL-6 were also positively correlated with AKI; however, the lymphocyte count and eGFR at admission were negatively related to AKI $(\mathrm{P}<0.05$; Table 5).

\section{Treatment}

During hospitalization, the patients received various treatments including antiviral therapy (90/96), oxygen therapy (76/96), traditional Chinese medicine (87/96), antibiotic treatment (49/96), anticoagulation therapy (18/96), glucocorticoids (8/96), antifungal treatment (5/96), continuous renal replacement therapy (CRRT) (4/96), intravenous immunoglobulin therapy (3/96), and mechanical ventilation (3/96). The 3 patients who required mechanical ventilation died due to respiratory failure, and 1 of them developed AKI and received CRRT. A dual plasma molecular adsorption system (DPMAS), hemodiafiltration, and hemoperfusion were applied in CRRT. Anticoagulation methods included unfractionated heparin, low molecular heparin, no heparin, regional citrate anticoagulation, and low-dose argatroban, and were given according to the different conditions of the patients. Of the 4 patients who underwent CRRT, 2 were diagnosed with AKI, and 2 suffered from cytokine storm with an elevated IL-6 level (up to $223.30 \mathrm{pg} / \mathrm{mL}$ ); unfortunately, none of these 4 patients survived. The patient with AKI who had a CKD history died of respiratory and circulatory failure, despite the use of antiviral therapy and other supportive treatments. Another patient with AKI, an 88-year old female without comorbidities, survived after effective treatment.

\section{Discussion}

COVID-19 has become a significant infectious disease involving rapid transmission and high mortality around 
Table 3 Laboratory results

\begin{tabular}{|c|c|c|c|c|}
\hline Variable & Total $(\mathrm{n}=96)$ & Severely ill $(n=61)$ & Critically ill $(n=35)$ & $P$ value \\
\hline White blood cell count $\left(\times 10^{9} / \mathrm{L}\right)$ & $6.0(4.6-7.2)$ & $5.9(4.8-6.9)$ & $6.1(4.2-8.8)$ & 0.523 \\
\hline Red blood cell count $\left(\times 10^{9} / \mathrm{L}\right)$ & $4.1(3.8-4.5)$ & $4.2(3.8-4.4)$ & $4.1(3.7-4.5)$ & 0.891 \\
\hline Hemoglobin (g/L) & $128[114-137]$ & 128 [114-135] & $128[114-138]$ & 0.840 \\
\hline Neutrophil count $\left(\times 10^{9} / \mathrm{L}\right)$ & $4.1(3.0-5.3)$ & $3.8(2.9-4.9)$ & $4.7(3.2-7.2)$ & 0.102 \\
\hline Lymphocyte count $\left(\times 10^{9} / \mathrm{L}\right)$ & $1.1(0.8-1.6)$ & $1.2(1.0-1.7)$ & $0.8(0.6-1.3)$ & $0.000^{\star *}$ \\
\hline Decreased lymphocyte (1.1-3.2), n (\%) & $48(50.0)$ & $23(37.7)$ & $25(71.4)$ & $0.001^{* *}$ \\
\hline \multicolumn{5}{|l|}{ Coagulation function } \\
\hline Fibrinogen (g/L) & $4.18(3.21-5.25)$ & $3.88(3.07-5.22)$ & $4.79(3.67-5.71)$ & $0.033^{*}$ \\
\hline Thrombin time (s) & $15.5(14.8-16.3)$ & $15.5(14.8-16.2)$ & $15.5(14.8-16.4)$ & 0.923 \\
\hline \multicolumn{5}{|l|}{ Blood biochemistry } \\
\hline Albumin (g/L) & $30.8(26.4-35.0)$ & $31.6(28.2-38.0)$ & $29.6(24.9-32.7)$ & $0.012^{*}$ \\
\hline Serum creatinine (umol/L) & $72.5(62.2-81.1)$ & $71.2(58.3-81.1)$ & $75.5(63.1-81.4)$ & 0.167 \\
\hline eGFR $\left(\mathrm{mL} / \mathrm{min} / 1.73 \mathrm{~m}^{2}\right)$ & $92.9(77.3-112.1)$ & $97.6(77.7-117.4)$ & $89.8(72.7-101.4)$ & 0.086 \\
\hline$A K I^{a}, n(\%)$ & $4(4.2)$ & $0(0.0)$ & $4(11.4)$ & 0.016 \\
\hline Total bilirubin (umol/L) & $11.0(8.6-14.1)$ & $10.7(8.3-13.4)$ & $12.0(9.6-18.2)$ & 0.077 \\
\hline Creatine kinase (U/L) & 58 [39-79] & 58 [45-73] & $55[29-154]$ & 0.741 \\
\hline A-hydroxybutyrate dehydrogenase (U/L) & 169 [138-213] & 151 [133-183] & $196[168-337]$ & $0.000^{* *}$ \\
\hline $\mathrm{TC}(\mathrm{mmol} / \mathrm{L})$ & $4.36(3.80-5.01)$ & $4.53(3.87-5.28)$ & $4.14(3.67-4.76)$ & 0.091 \\
\hline $\mathrm{TG}(\mathrm{mmol} / \mathrm{L})$ & $1.49(1.15-2.08)$ & $1.62(1.16-2.21)$ & $1.43(1.12-1.92)$ & 0.321 \\
\hline $\mathrm{HDL}(\mathrm{mmol} / \mathrm{L})$ & $0.96(0.81-1.22)$ & $1.08(0.86-1.26)$ & $0.87(0.74-1.06)$ & $0.003^{\star \star}$ \\
\hline LDL (mmol/L) & $2.60(1.98-3.22)$ & $2.63(2.10-3.39)$ & 2.49 (1.96-2.93) & 0.565 \\
\hline Potassium (mmol/L) & 3.87 (3.57-4.11) & $3.84(3.59-4.11)$ & 3.87 (3.56-4.09) & 0.861 \\
\hline Sodium (mmol/L) & 140 [137-142] & 140 [138-143] & 138 [134-141] & $0.019^{*}$ \\
\hline Calcium (mmol/L) & $1.96(1.90-2.06)$ & $1.98(1.91-2.08)$ & $1.94(1.87-2.06)$ & 0.341 \\
\hline PCT (ng/mL) & $0.06(0.04-0.11)$ & $0.05(0.04-0.08)$ & $0.10(0.06-0.24)$ & $0.000^{\star *}$ \\
\hline CRP (mg/L) & $6.36(1.46-54.20)$ & $3.82(0.31-13.56)$ & $56.40(12.33-89.29)$ & $0.000^{\star \star *}$ \\
\hline
\end{tabular}

Table 3 (continued) 
Table 3 (continued)

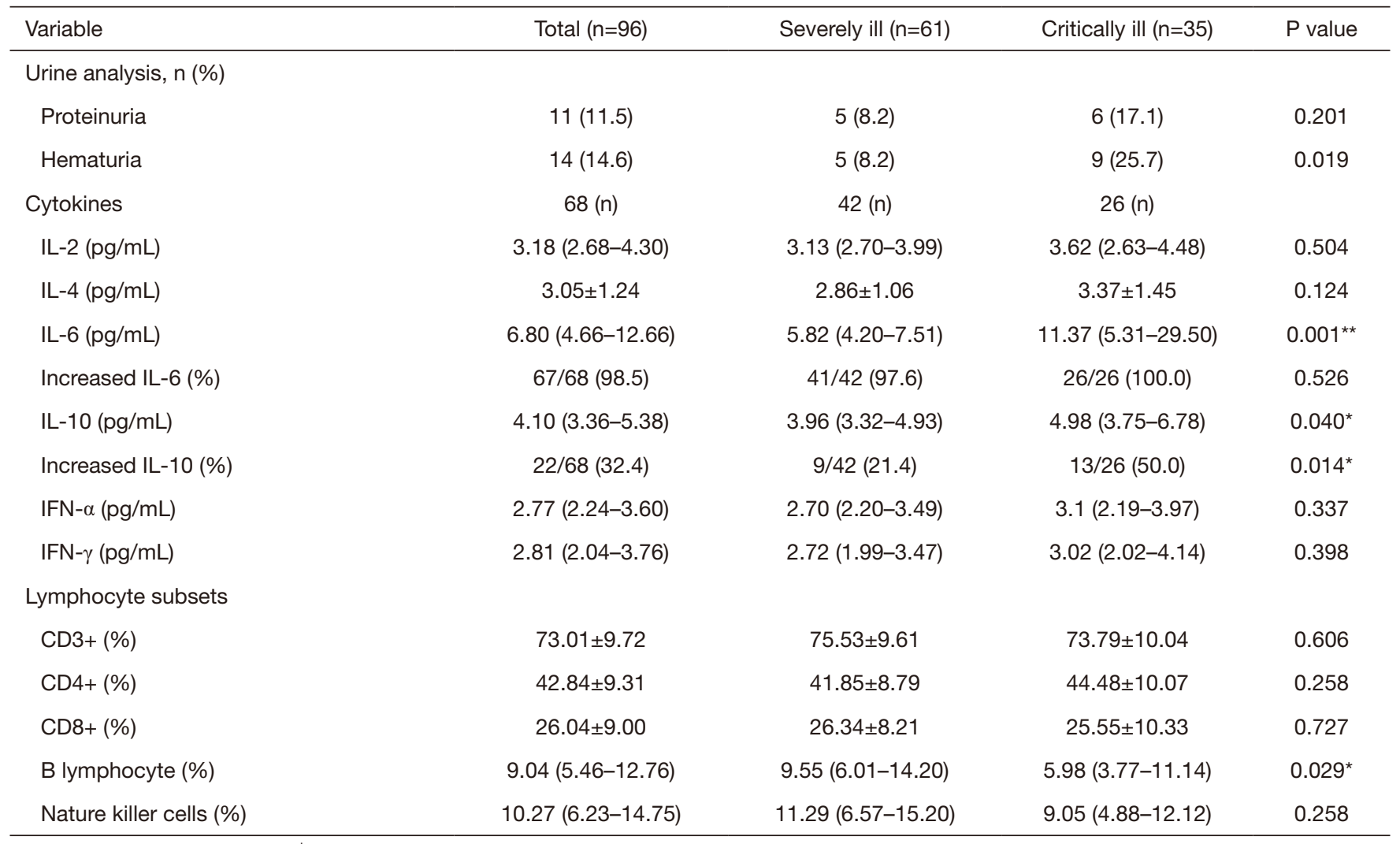

a Using the fisher exact test; ${ }^{b} \mathrm{AST}>40 \mathrm{U} / \mathrm{L} ; \mathrm{LDH}>245 \mathrm{U} / \mathrm{L} ;{ }^{*} \mathrm{P}<0.05 ;{ }^{* \star} \mathrm{P} \leq 0.01$. eGFR, estimated glomerular filtration rate; AKI, acute kidney injury; TC, total cholesterol; TG, triglyceride; HDL, high density lipoprotein; LDL, low density lipoprotein; PCT, procalcitonin; CRP, C-reactive protein; IL, interleukin; IFN, interferon.

Table 4 Detection of SARS-CoV-2

\begin{tabular}{|c|c|c|c|c|}
\hline Variable & Total $(n=96)$ & Severely ill $(n=61)$ & Critically ill $(n=35)$ & $P$ value \\
\hline Negative conversion rate ${ }^{a}(\%)$ & $95(99.0)$ & $61(100.0)$ & $34(97.1)$ & 0.365 \\
\hline Days from positive to negative laboratory test & $17(13-22)$ & $17(13-23)$ & $17(12-21)$ & 0.630 \\
\hline Days from symptoms to negative laboratory test & $27(23-34)$ & $27(23-35)$ & $26(24-34)$ & 0.978 \\
\hline False negative rate $(\%)$ & $27(28.1)$ & $14(23.0)$ & $13(37.1)$ & 0.137 \\
\hline
\end{tabular}

${ }^{a}$ Using the Fisher exact test.

Table 5 The variables related to AKI by spearman correlation analysis

\begin{tabular}{lclcllcc}
\hline AKI & Condition & Age & Lymphocyte & CRP & PCT & eGFR \\
\hline$r_{\mathrm{s}}$ & 0.275 & 0.213 & -0.207 & 0.255 & 0.266 & -0.237 \\
$\mathrm{P}$ value & $0.007^{\star *}$ & $0.037^{*}$ & $0.043^{*}$ & $0.012^{*}$ & $0.010^{* *}$ & $0.020^{\star}$ & $0.011^{*}$ \\
\hline
\end{tabular}

${ }^{*} \mathrm{P}<0.05 ;{ }^{*} \mathrm{P} \leq 0.01$. AKI, acute kidney injury; $r_{\mathrm{s}}$, correlation coefficient; CRP, C-reactive protein; PCT, procalcitonin; eGFR, estimated glomerular filtration rate; IL-6, interleukin-6. 
the globe $(2,3)$. In our study, we showed that patients with COVID-19 demonstrated elevated levels of D-dimers, fibrinogen, PCT, and IL-6, as well as increased hypoalbuminemia, hypocalcemia, and lymphopenia. Five meta-analyses including more than 20,000 patients indicated the incidence of AKI among patients with COVID-19 to range from $4.5 \%$ to $18 \%$ (9). Our data showed that the prevalence of AKI in the entire study population and in patients without a CKD history was $4.2 \%$ and $3.3 \%$, respectively, which was slightly lower than the incidence reported in existing reports (10). We speculate that this difference may be due to the fact that in other research, most patients had intrinsic kidney damage, whereas our patients did not. Several studies have shown that patients with COVID-19 who developed AKI had longer hospitalization durations, were more likely to be admitted to an ICU, and had a higher mortality rate (9). In our study, the fact that the 4 patients who developed AKI were all from the critically ill group and that 3 of them died also supports these findings. Moreover, we proved that in severely and critically ill COVID-19 patients, age, disease severity, PCT, CRP, and IL-6 were correlated with AKI, whereas lymphocyte count and eGFR at admission were inversely related to the development of AKI. Taken together, these findings provide insights into the prevention of AKI in patients with COVID-19.

We hypothesize that diastolic pressure reduction, hypercoagulability, and cytokine damage may be related to renal damage in patients with COVID-19. Studies have reported diastolic perfusion pressure to be independently associated with the development of cardiac surgeryassociated AKI, which is related to blood perfusion in the kidney (11). Moreover, since angiotensin-converting enzyme 2 (ACE2) is highly expressed in renal tubular cells, SARS-CoV-2 might directly bind to ACE2-positive cells, thereby disturbing the regulation of normal blood pressure and ultimately damaging the kidney (12). Secondly, thrombocytopenia is independently associated with multiple organ failure including AKI (13). Thirdly, cytokines, especially IL-6, can induce intrarenal inflammation and result in AKI. Ronco et al. also reported organ crosstalk and systemic effects as potential mechanisms of kidney involvement (14). In the present study, further comparison between severely and critically ill cases revealed that critically ill patients were older, had a lower diastolic pressure, and more severe polypnea, lymphopenia, and hypercoagulability. Moreover, their liver and renal dysfunction as well as immunoinflammatory responses were significantly worse than those of severely ill patients. Accumulating evidence suggests that a subgroup of patients with severe COVID-19 might suffer from a cytokine storm syndrome (15-17), which is in accordance with our findings. Indeed, relationships can be identified from the abovementioned findings. A study at the Peking Union Medical College Hospital suggested that immune system disorder in patients with COVID-19 is related to hypercoagulability, which is part of the cytokine storm and may increase the risk of autoimmune disease later in life (18). Another study indicated that the decrease in diastolic pressure can be interpreted as a result of hypercoagulability and ACE2 binding to the virus, which leads to disorders of blood pressure regulation (12). These findings illustrate that critically ill patients might have been more susceptible to AKI than severely ill patients in our study.

For the patients in our study, arbidol, ribavirin, and oseltamivir were used as antiviral therapies. To date, there have been no reports on arbidol- or oseltamivir-induced AKI, and only 1 study has reported severe hyperuricemia and AKI developing early after the initiation of ribavirin for respiratory syncytial virus infection (19). Therefore, the correlation between ribavirin and AKI is unclear. The effects of antiviral drugs on kidney function need to be observed in more studies in future. Blood purification therapy may be effective in reducing the cytokine storm in critically ill patients, and early control of the cytokine storm might be beneficial to survival and kidney recovery (20). On April $10^{\text {th }}, 2020$, the United States Food and Drug Administration (FDA) issued an emergency-use authorization for blood purification for confirmed COVID-19 patients aged 18 years and over who have respiratory failure or are about to develop respiratory failure. The FDA has also approved the Spectra Optia adsorption system and Depuro D2000 adsorption cartridge for plasma exchange and cytokine clearance. Although blood purification therapy may be effective, the outcomes of the AKI patients who underwent CRRT in our study did not improve; therefore, additional studies are warranted.

This study has limitations. For instance, it is a singlecenter study with limited case numbers; thus, further studies are required to verify our results.

In conclusion, our findings demonstrate that AKI is not common in severely and critically ill COVID-19 patients without a history of CKD. Compared with severely ill patients, critically ill patients are more susceptible to developing AKI. Age, disease severity, PCT, CRP, IL-6, lymphocyte count, and eGFR are all correlated with AKI 
onset. Although blood purification therapy may be effective, the outcomes of AKI patients who underwent CRRT in our study did not improve.

\section{Acknowledgments}

The authors would like to thank the pathologists for their assistance. We would like to thank C. Betlazar-Maseh and J. Reynolds for their help in polishing our paper.

Funding: This work was supported by the National Natural Science Foundation of China (No. 81673499) and Natural Science Foundation of Hunan Province (No. 2017SK2051, No. 2018JJ3835).

\section{Footnote}

Reporting Checklist: The authors have completed the STROBE reporting checklist. Available at http://dx.doi. org/10.21037/apm-20-2170

Data Sharing Statement: Available at http://dx.doi. org/10.21037/apm-20-2170

Conflicts of Interest: All authors have completed the ICMJE uniform disclosure form (available at http://dx.doi. org/10.21037/apm-20-2170). ZP reports that she receives funding from the National Natural Science Foundation of China and Natural Science Foundation of Hunan Province. The other authors have no conflicts of interest to declare.

Ethical Statement: The authors are accountable for all aspects of the work in ensuring that questions related to the accuracy or integrity of any part of the work are appropriately investigated and resolved. This study was approved by the ethics committee of Xiangya Hospital, China(No. 202003049), and was in adherence with the Declaration of Helsinki (as was revised in 2013). Informed consent was obtained from the patients before data collection.

Open Access Statement: This is an Open Access article distributed in accordance with the Creative Commons Attribution-NonCommercial-NoDerivs 4.0 International License (CC BY-NC-ND 4.0), which permits the noncommercial replication and distribution of the article with the strict proviso that no changes or edits are made and the original work is properly cited (including links to both the formal publication through the relevant DOI and the license).
See: https://creativecommons.org/licenses/by-nc-nd/4.0/.

\section{References}

1. Li Q, Guan X, Wu P, et al. Early transmission dynamics in Wuhan, China, of novel coronavirus-infected pneumonia. N Engl J Med 2020;382:1199-207.

2. Zhou F, Yu T, Du R, et al. Clinical course and risk factors for mortality of adult inpatients with COVID-19 in Wuhan, China: a retrospective cohort study. Lancet 2020;395:1054-62.

3. Huang C, Wang Y, Li X, et al. Clinical features of patients infected with 2019 novel coronavirus in Wuhan, China. Lancet 2020;395:497-506.

4. Li Z, Wu M, Guo J, et al. Caution on Kidney Dysfunctions of 2019-nCoV Patients. medRxiv preprint doi: https://doi. org/10.1101/2020.02.08.20021212.

5. Wang L, Li X, Chen H, et al. Coronavirus Disease 19 Infection Does Not Result in Acute Kidney Injury: An Analysis of 116 Hospitalized Patients from Wuhan, China. Am J Nephrol 2020;51:343-8.

6. Gabarre P, Dumas G, Dupont T, et al. Acute kidney injury in critically ill patients with COVID-19. Intensive Care Med 2020;46:1339-48.

7. Levey AS, Stevens LA, Schmid CH, et al. CKD-EPI (Chronic Kidney Disease Epidemiology Collaboration). A new equation to estimate glomerular filtration rate. Ann Intern Med 2009;150:604-12.

8. Khwaja A. KDIGO clinical practice guidelines for acute kidney injury. Nephron Clin Pract 2012;120:c179-84.

9. Sanchez-Russo L, Billah M, Chancay J, et al. COVID-19 and the Kidney: A Worrisome Scenario of Acute and Chronic Consequences. J Clin Med 2021;10:900.

10. Cheng Y, Luo R, Wang K, et al. Kidney disease is associated with in-hospital death of patients with COVID-19. Kidney Int 2020;97:829-38.

11. Jin J, Yu J, Chang SC, et al. Postoperative diastolic perfusion pressure is associated with the development of acute kidney injury in patients after cardiac surgery: a retrospective analysis. BMC Nephrol 2019;20:458.

12. Caibin Fan, Kai Li, Yanhong Ding, et al. ACE2 Expression in Kidney and Testis May Cause Kidney and Testis Damage After 2019-nCoV Infection. medRxiv preprint doi: https://doi.org/10.1101/2020.02.12.20022 418.

13. Nguyen TC, Cruz MA, Carcillo JA. Thrombocytopenia-Associated Multiple Organ Failure and Acute Kidney Injury. Crit Care Clin 2015;31:661-74. 
14. Ronco C, Reis T. Kidney involvement in COVID-19 and rationale for extracorporeal therapies. Nat Rev Nephrol 2020;16:308-10.

15. Mehta P, McAuley DF, Brown M, et al. COVID-19: consider cytokine storm syndromes and immunosuppression. Lancet 2020;395:1033-4.

16. Vaninov N. In the eye of the COVID-19 cytokine storm. Nat Rev Immunol 2020;20:277.

17. Yang Y, Shen C, Li J, et al. Exuberant elevation of IP10, MCP-3 and IL-1ra during SARS-CoV-2 infection is associated with disease severity and fatal outcome. Preprint at medRxiv https://doi.org/10.1101/2020.03.02.20029975

Cite this article as: $\mathrm{Yu} Y$, Ge H, Wang X, Huang Z, Chen L, Han Y, Jiang D, Tao L, Zhou Q, Peng Z, Xiao X. A survey on acute kidney injury in severely and critically ill COVID-19 patients without chronic kidney disease. Ann Palliat Med 2021;10(6):6198-6207. doi: 10.21037/apm-20-2170
(2020).

18. Zhang Y, Xiao M, Zhang S, et al. Coagulopathy and Antiphospholipid Antibodies in Patients with Covid-19. N Engl J Med 2020;382:e38.

19. Sakran R, Frisch A, Elias A, et al. Acute and severe ribavirin-associated hyperuricemia and acute kidney injury: An underrecognized adverse effect. Am J Health Syst Pharm 2021;22:zxab043.

20. Ma J, Xia P, Zhou Y, et al. Potential effect of blood purification therapy in reducing cytokine storm as a late complication of critically ill COVID-19. Clin Immunol 2020;214:108408. Clin Immunol 2020;214:108408. 